\title{
Effect of Codonopsis pilosula Polysaccharides on the Growth and Motility of Hepatocellular Carcinoma HepG2 Cells by Regulating $\beta$-Catenin/TCF4 Pathway
}

\author{
Yuan-yuan Zhang $\mathbb{D}^{1}{ }^{1}$ Ying-mei Zhang, ${ }^{1}$ and Hai-yan $\mathrm{Xu} \mathbb{D}^{2}$ \\ ${ }^{1}$ Department of Laboratory Medicine, The Affiliated Huaian No. 1 People's Hospital of Nanjing Medical University, Huai'an, \\ Jiangsu 223300, China \\ ${ }^{2}$ Department of Cardiology, The Affiliated Huaian No. 1 People's Hospital of Nanjing Medical University, Huai'an, \\ Jiangsu 223300, China
}

Correspondence should be addressed to Yuan-yuan Zhang; yuanyuanzhang1234@163.com

Received 11 March 2019; Revised 12 April 2019; Accepted 15 April 2019; Published 8 May 2019

Guest Editor: Jianxun Ding

Copyright (c) 2019 Yuan-yuan Zhang et al. This is an open access article distributed under the Creative Commons Attribution License, which permits unrestricted use, distribution, and reproduction in any medium, provided the original work is properly cited.

\begin{abstract}
Objective. To study the effect of Codonopsis pilosula polysaccharide (CPP) on the growth and motility of HepG2 cells and its possible mechanism. Methods. Cells were randomly divided into Control group, CPP $(5 \mu \mathrm{M})$ group, CPP $(10 \mu \mathrm{M})$ group, and CPP $(20 \mu \mathrm{M})$ group. The proliferation, invasion, migration ability, and expression of proteins involved in the epithelial-mesenchymal transition (EMT) and signaling pathway of HepG2 cells were detected by CCK8 assay, BrdU staining, Transwell, Scratch test, and Western blot, respectively. Results. Codonopsis pilosula polysaccharide inhibited the proliferation of HepG2 cells cultured in vitro along with the expression level of Ki67 and PCNA protein $(P<0.05)$, decreased the number of invasive cells $(P<0.05)$, and reduced the scratch closure rate $(P<0.05)$. It also adjusted the expression of vascular endothelial growth factor (VEGF), E-cadherin, and N-cadherin $(P<0.05)$. Other than that, downregulation of $\beta$-catenin, TCF4, and $\mathrm{c}$-Myc protein expression $(P<0.05)$ was observed as well. Conclusion. Codonopsis pilosula polysaccharide can inhibit the proliferation and motility of HepG2 cells cultured in vitro, and the underlying mechanism is proposed to be related to the inhibition of the $\beta$-catenin/TCF4 pathway.
\end{abstract}

\section{Introduction}

Hepatocellular carcinoma (HCC) is one of the leading causes of cancer-related mortality worldwide. The main driving factors associated with HCC include chronic infection of hepatitis B and chronic hepatitis $\mathrm{C}$, alcoholic liver disease, and nonalcoholic fatty liver disease. Despite advances in new technologies of prevention, screening, diagnosis, and treatment of HCC, the morbidity and mortality rate of the disease continue to rise. About 600,000 people die of liver cancer every year worldwide. The treatments vary considering different tumor load and metastasis; moreover, the treatment for late-stage liver cancer is expensive and ineffective $[1-3]$. Therefore, it is necessary to expand researches regarding the treatment of HCC and identify more effective therapeutic drugs. Codonopsis pilosula, also known as dangshen, belongs to the Campanulaceae family. The dried root from its subspecies dangshen, suhuadangshen, or chuandangshen, containing polysaccharides, saponins, sesquiterpenoids, polyphenols, terpenoids, alkaloids, volatile oil, and other ingredients, is commonly prepared for medical use. Codonopsis pilosula polysaccharide (CPP), one of active constituents in Codonopsis pilosula, has attracted extensive attention in the medical field due to its extensive physiologi$\mathrm{cal}$ and biological activities. Previous studies have shown that Codonopsis pilosula polysaccharide has the effects of immunity enhancement, antivirus, liver protection, and neuroprotection [4-9]. It is worth noting that the polysaccharide from Codonopsis pilosula plays an important role in growth inhibition in various tumor cells such as breast cancer and 
cervical cancer $[10,11]$. At present, however, there are few reports on the effect of Codonopsis pilosula polysaccharide on human hepatoma HepG2 cells. Taken all these together, in this study, we studied the human hepatoma HepG2 cells cultured in vitro and explored the effect of Codonopsis pilosula polysaccharide on the proliferation and metastasis of hepatocarcinoma cells and its possible mechanism as well.

\section{Materials and Methods}

2.1. Reagents and Instruments. Dangshen polysaccharide (purity $\geq 98 \%$ ) was purchased from China Food and Drug Administration Research Institute. DMEM cell culture medium, $0.25 \%$ trypsin, and fetal bovine serum were purchased from Gibco, USA. The CCK8 kit was purchased from Roche Group. Transwell chamber was purchased from Beijing Unicom Biotechnology Co. LTD. Monoclonal antibody and horseradish peroxidase-labeled secondary antibody were purchased from Abcam, UK. RIPA lysate was purchased from Sigma, USA. The BCA kit was purchased from Biyuntian Biotech. BrdU detection kits were purchased from Guangzhou Ruibo Biological Co. Ltd.

Both the electrophoresis apparatus and the semidry film transfer apparatus were purchased from Bole Corporation of the United States. The Gel View 6000 chemiluminescent gel imaging system was purchased from Guangzhou Yunxing Instrument Co. Ltd. The ordinary optical microscope was purchased from Olympus Corporation of Japan.

2.2. Cell Culture. The human liver cancer HepG2 cell line was purchased from FuHeng Cell Center, Shanghai, China. The cells were cultured in a DMEM medium containing 10\% fetal bovine serum and $1 \%$ cyan-streptomycin at $37^{\circ} \mathrm{C}$ in a $5 \%$ $\mathrm{CO}_{2}$ incubator and subcultured when the cell fusion rate reached $80 \%$.

\subsection{Experimental Methods}

2.3.1. Cell Activity Was Detected by CCK8 Assay. The cells were seeded in a 96-well plate at a density of $4 \times 104$ cells $/ \mathrm{mL}$. After cell attachment, the cells were treated with different concentrations of CPP $(0,0.05,0.1,0.2,0.5,1,2,5,10,20$, $50,100,200$, and $400 \mu \mathrm{M})$ for $24 \mathrm{~h}$, with 6 replicate wells per concentration. After $24 \mathrm{~h}, 10 \mu \mathrm{L}$ of CCK8 reagent was added into each well and after incubation at $37^{\circ} \mathrm{C}$ for $4 \mathrm{~h}$; the activity of each well was measured by an enzymelabeling instrument. According to the test results, the CPP concentrations resulting in over $80 \%$ of cell activity were selected for subsequent experiments.

2.3.2. BrdU Staining for Cell Proliferation. The cells were seeded in a 6-well plate with $1 \mathrm{~mL}$ of cell suspension at the density of $2 \times 10^{6}$ cells $/ \mathrm{mL}$ for each well. After cell attachment, the cells were incubated in a medium containing $0.4 \%$ FBS for 72 hours, each group containing 3 replicate wells. The cells were randomly divided into 4 groups: Control group, CPP $(5 \mu \mathrm{M})$ group, CPP $(10 \mu \mathrm{M})$ group, and CPP $(20 \mu \mathrm{M})$ group. After the corresponding treatment of final concentration liquor, BrdU with final concentration of $0.03 \mu \mathrm{g} / \mathrm{mL}$ was added before another 40 minutes of incubation. The culture medium was discarded, and the plate was washed three times with PBS, fixed with paraformaldehyde for $10 \mathrm{~min}$, and cell proliferation assay was performed strictly according to the instructions. The number of BrdUpositive cells was counted under a microscope.

2.3.3. Transwell Detection of Cell Invasion Ability. The cells were seeded into the upper chamber of Matrigel-coated Transwell chambers at a density of $4 \times 10^{5}$ cells $/ \mathrm{mL}$, cultured in a medium containing no fetal bovine serum, while at the lower chamber, normal cell culture medium was added. After grouping according to Section 2.3.1 and further culture for 48 hours, the cells in the upper chamber were wiped with a sterile cotton swab, and the cells migrated to the lower chamber were stained with crystal violet. Five fields were randomly selected for each group for counting. There were 6 replicate wells in each group.

2.3.4. Cell Migration Ability Was Detected by Scratch Test. Cells were seeded in 12 -well sterile plates at a density of $4 \times 10^{5}$ cells $/ \mathrm{mL}$. After attachment of cells to the wall, horizontal lines perpendicular to the marker were drawn by the $10 \mu \mathrm{L}$ gun tip, and the plate was washed 3 times with PBS. After grouping according to Section 2.3.1 and drug administering, the plate was further cultured for $24 \mathrm{~h}$. Wound closure rate $=($ initial width - measurement width)/initial width $\times 100 \%$.

2.3.5. Western Blot Detection of Cell Proliferation, EMT, and Pathway Protein Expression. The total protein of each group was extracted with RIPA protein lysate on ice, and the concentration of proteins was measured and leveled by BCA kit for each group. Equal amount of proteins for each group was separated by $12 \%$ SDS-PAGE and transferred into a PVDF membrane. The membrane was then blocked with $5 \%$ skim milk for $2 \mathrm{~h}$ at room temperature. The blot was incubated in primary antibody overnight at $4^{\circ} \mathrm{C}$. The next day, the primary antibody was discarded, and the bufferwashed PVDF blot was incubated in corresponding secondary antibody for 1 hour at room temperature. For imaging, ECL solution was added dropwise in the dark room for blot exposure and development. In this study, GAPDH was used as the internal reference.

2.4. Statistical Methods. All experimental data were statistically analyzed with statistical software SPSS 19.0. Differences between groups were tested by $t$, $t$-test, or one-way ANOVA. The experimental results were expressed as mean \pm standard deviation. The difference was considered statistically significant at $P<0.05$.

\section{Results}

3.1. Effect of CPP on the Proliferation of HepG2 Cells Cultured In Vitro. The effect of different concentrations of CPP on the survival rate of HepG2 cells was measured by CCK8 assay, as shown in Figure 1. As the CPP concentration reaches $50 \mu \mathrm{M}$ and above, the survival rate of HepG2 cells remains less than $80 \%$. It indicated that CPP concentration up to $50 \mu \mathrm{M}$ has obvious cytotoxic effect on HepG2 


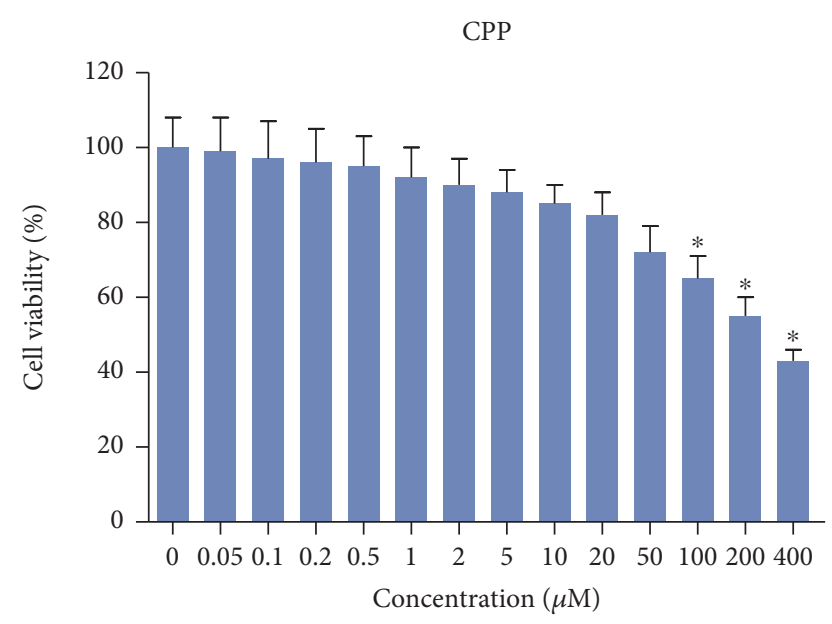

Figure 1: Cell viability of HepG2 cells.

cells. Therefore, we selected 3 maximum concentrations of 5,10 , and $20 \mu \mathrm{M}$ without a significant cytotoxicity for subsequent experiments.

The proliferation of cells was detected by BrdU staining. The results showed that the number of BrdU-positive cells in the CPP-treated groups was significantly lower than that in the Control group $(P<0.05$, Figure 2(a)) and inversely proportional to the concentration of CPP. At the same time, the expression levels of proliferation-related proteins were further detected by Western blot. The results showed that the expression levels of Ki67 and PCNA proteins in CPPtreated cells were significantly lower than those in the Control group $(P<0.05$, Figure $2(b))$, and the differences were also CPP concentration-dependent.

3.2. Effect of CPP on the Invasive Ability of HepG2 Cells Cultured In Vitro. The cell invasive ability was determined by Transwell assay, and the results showed that, compared with the Control group, the CPP-treated groups showed significantly reduced number of invasive cells $(P<0.05$, Figure 3); moreover, the number decreased with the increase of CPP treatment concentration.

3.3. Effect of CPP on the Migration Ability of HepG2 Cells Cultured In Vitro. The cell migration ability was tested by the scratch test. The results suggested that the wound closure rate of the CPP-treated groups was significantly lower than that of the Control group $(P<0.05$, Figure 4$)$ and was CPP concentration-dependent.

3.4. Effect of CPP on the Expression of EMT-Related Protein in HepG2 Cells Cultured In Vitro. The morphology of the cells in each group was observed under the microscope. The cells in the Control group were mostly ovoid in shape or nearly square with tight arrangement. While after CPP treatment, the cells gradually displayed long fusiform with loose arrangement.

Western blot results represented the expression level of EMT-related protein in cells. The results showed that the expression levels of VEGF and N-cadherin were significantly decreased in CPP-treated groups compared with the Control group $(P<0.05$, Figure 5). In contrary, the expression level of E-cadherin protein was significantly upregulated $(P<0.05$, Figure 4) and was CPP concentration-dependent.

3.5. Effect of CPP on the Expression of Pathway Protein in HepG2 Cells Cultured In Vitro. The expression level of $\beta$-catenin, TCF4, and c-Myc proteins in CPP-treated cells was significantly lower than that in the Control group $(P<0.05$, Figure 6) and was positively correlated with the CCP treatment concentration.

\section{Discussion}

Hepatocellular carcinoma is a primary liver cancer to which most liver cancers belong. As a malignant tumor originating from the liver epithelial or mesenchymal tissue, it is prone to vascular invasion and metastasis, which mainly develop in the portal vein or its branches, hepatic veins or its branches, and the inferior vena cava of the liver, and homotype clumping in the portal vein is the most common form of metastasis of hepatocellular carcinoma [2-12]. The etiology and exact molecular mechanism are still unclear, and it is currently considered to be a multifactor and multistep complex process. The treatment method is specialized for each individual and the comprehensive treatment depends on the different stages of the disease. The treatment types mainly include surgery, hepatic artery ligation, radiofrequency, freezing, laser, microwave, and chemotherapy. Many studies have shown that Codonopsis pilosula polysaccharides have a variety of biological functions, including cardiovascular and cerebrovascular protection, enhancing immunity and antitumor activities [4-11]. Based on all facts above, we studied human hepatoma HepG2 cells and further investigated the effect of Codonopsis pilosula polysaccharides on the proliferation and metastasis ability of hepatoma cells in vitro and its possible mechanism.

Firstly, the effect of Codonopsis pilosula polysaccharide on the proliferation of HepG2 cells in vitro was detected by BrdU staining. It was found that Codonopsis pilosula polysaccharide dose-dependently reduced the number of BrdU-positive cells in HepG2 cells. The effect of Codonopsis pilosula polysaccharides on the expression levels of proliferation-related proteins Ki67 and PCNA in hepatocellular carcinoma HepG2 cells was further examined by Western blot experiments. It was confirmed that Codonopsis pilosula polysaccharide dose-dependently inhibited the expression levels of proliferation proteins. These results complied with that of Xin et al.'s study on the effect of Codonopsis pilosula polysaccharide on ovarian cancer HO-8910 cells [11]. On the other hand, Bai et al.'s study [13] showed that the two water-soluble polysaccharides extracted from Codonopsis pilosula polysaccharide can inhibit the growth of HepG2 cells, and the underlying mechanism is related to cell cycle arrest induction and key protein expression inhibition.

In order to test the effect of Codonopsis pilosula polysaccharide on the motility of hepatocellular carcinoma HepG2 cells cultured in vitro, we performed a scratch test and a Transwell experiment, both of which demonstrated the 

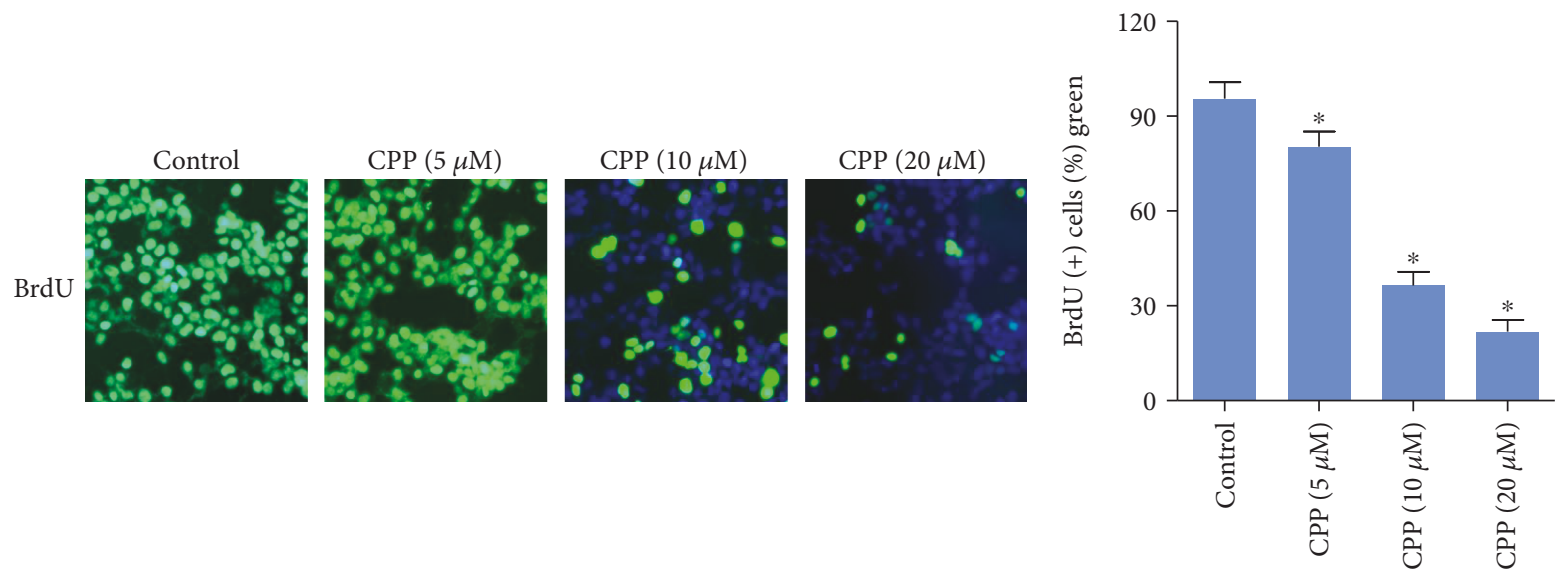

(a)

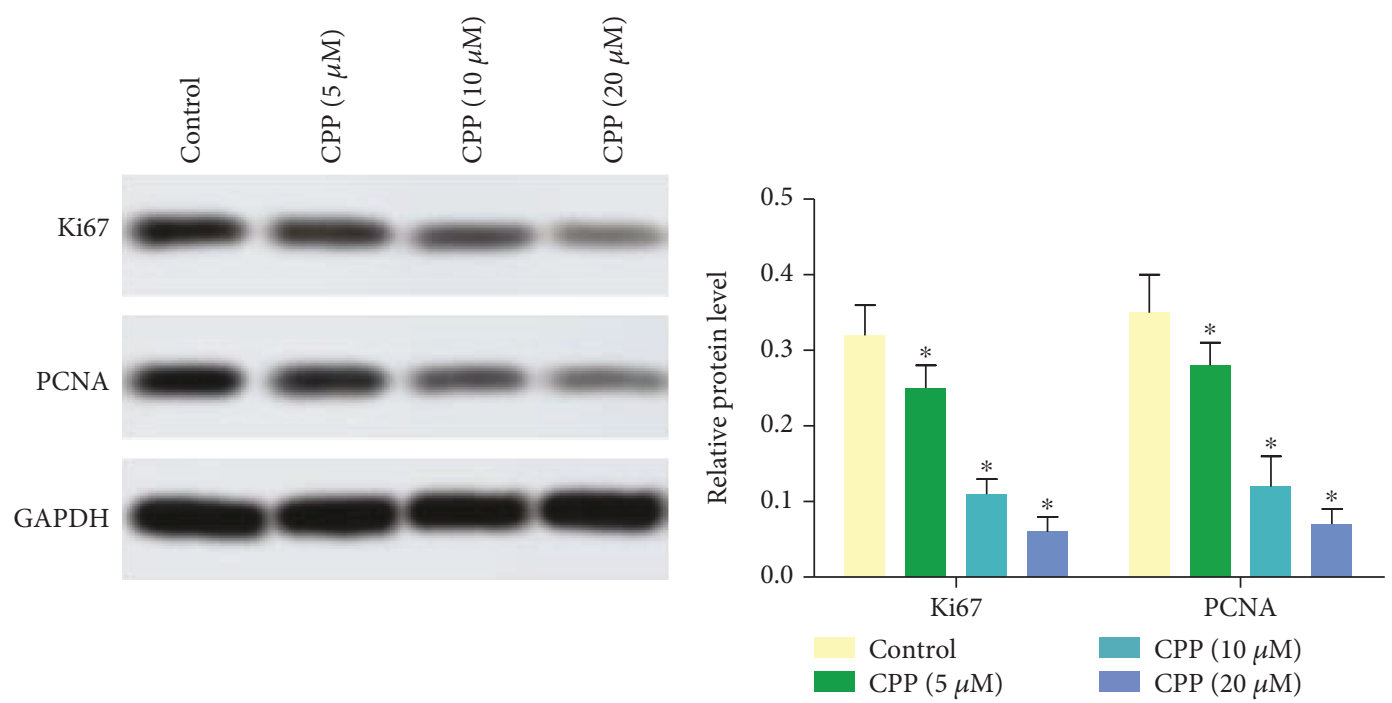

(b)

Figure 2: Proliferation of HepG2 cells. (a) Cell proliferation detected by BrdU; (b) protein expression of Ki67 and PCNA was tested by Western blot. ${ }^{*} P<0.05$ versus Control group.
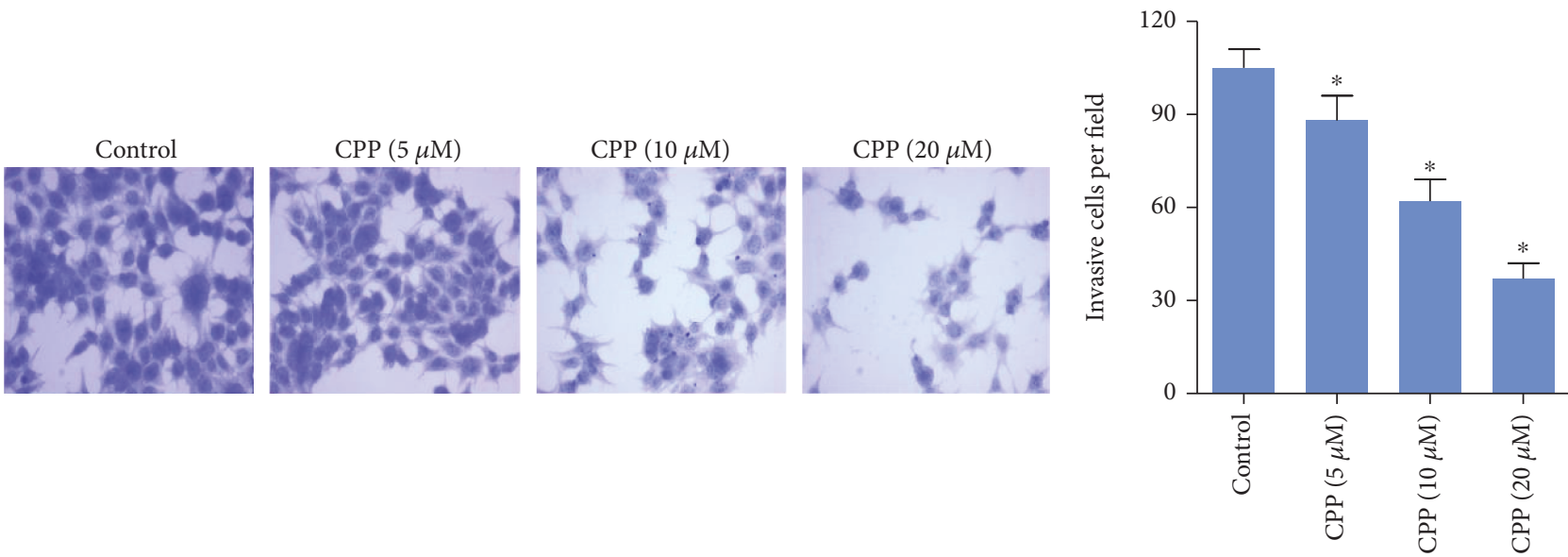

Figure 3: Invasion of HepG2 cells. ${ }^{*} P<0.05$ versus Control group. 


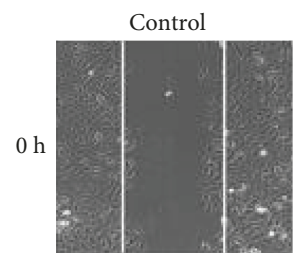

$\mathrm{CPP}(5 \mu \mathrm{M})$
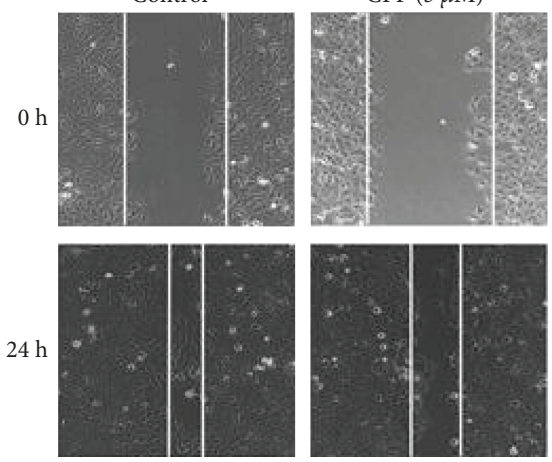

$\mathrm{CPP}(10 \mu \mathrm{M})$
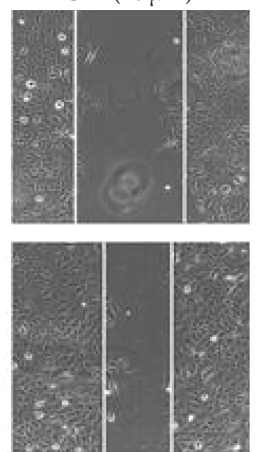

$\mathrm{CPP}(20 \mu \mathrm{M})$
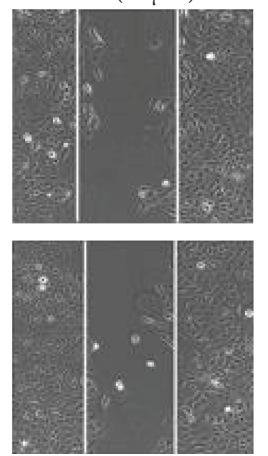

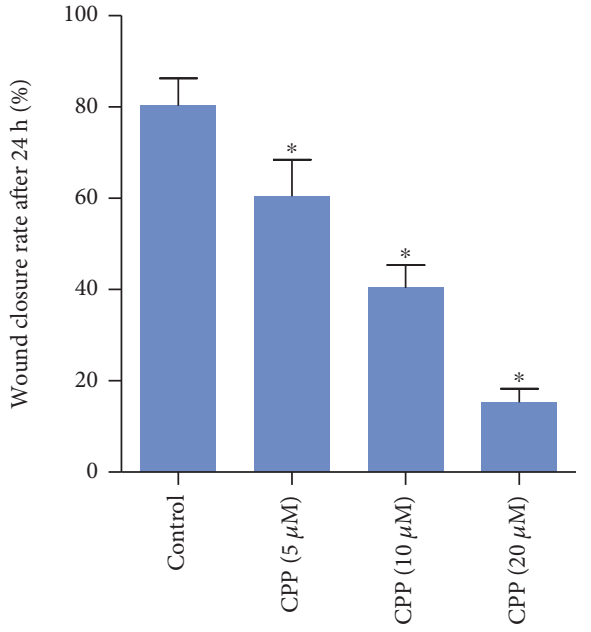

Figure 4: Migration of HepG2 cells. ${ }^{*} P<0.05$ versus Control group.
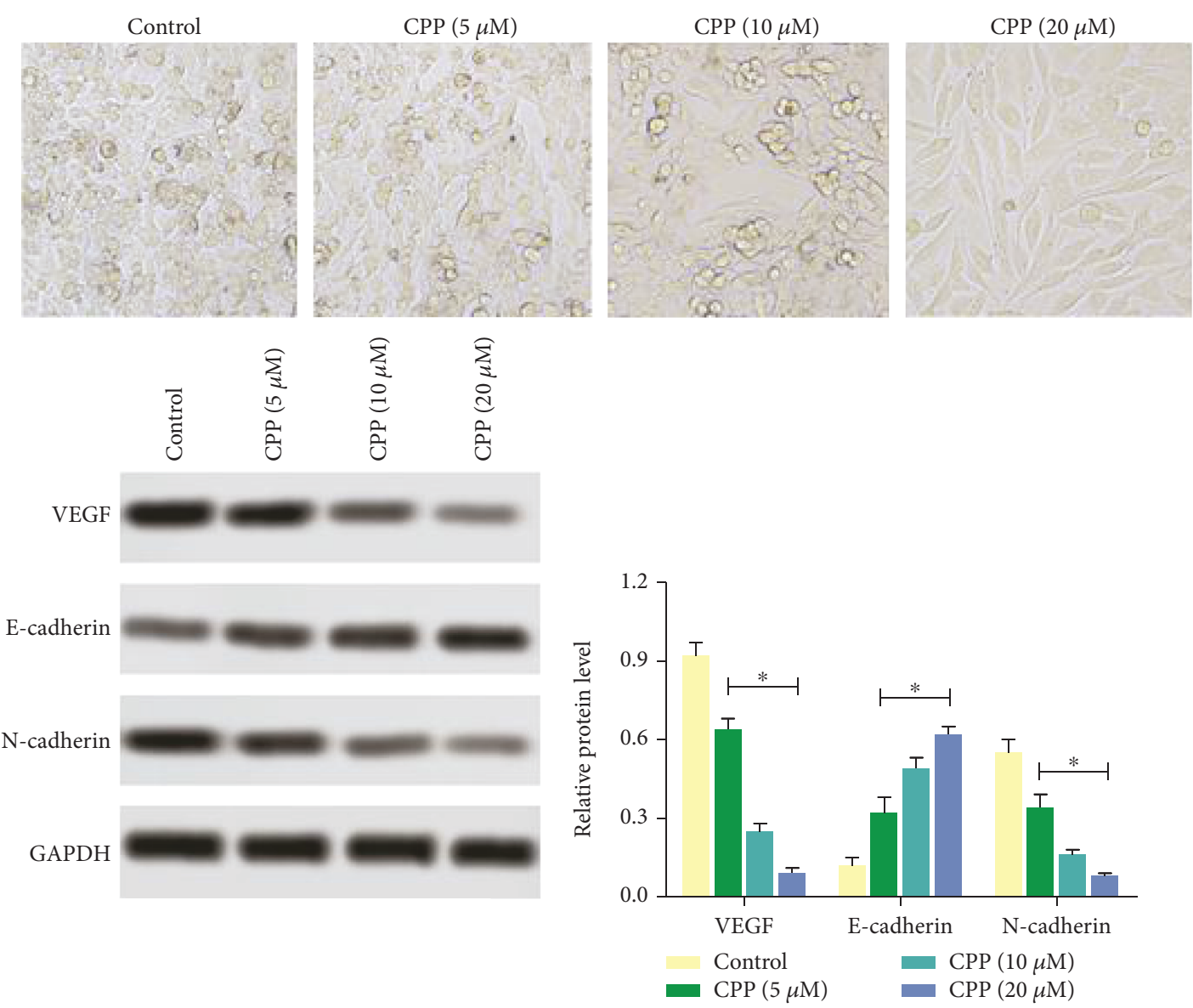

Figure 5: Expression of EMT-related protein in HepG2 cells. ${ }^{*} P<0.05$ versus Control group.

inhibitory effect of Codonopsis pilosula polysaccharide on the motility of HepG2 cells cultured in vitro. All these results are consistent with Bai et al.'s study [13].

Increased tumor cell migration capacity is the basis for tissue infiltration and distant metastasis. Epithelial mesenchymal transition (EMT) is a process in which polarized epithelial cells lose epithelial properties and acquire interstitial properties, thus increasing cell metastasis and invasion; it is an important biological process that promotes tumor invasion and metastasis [14, 15]. E-cadherin, as an 


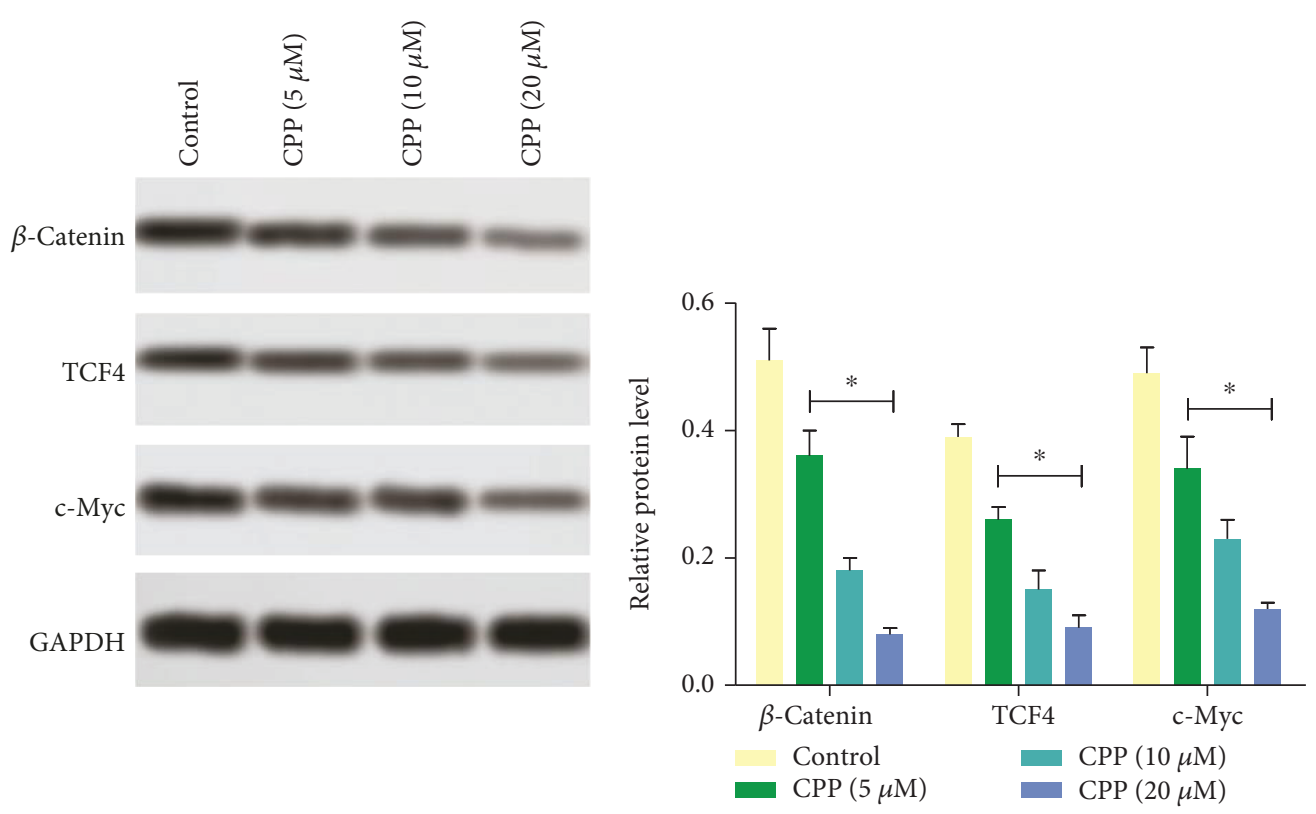

FIgURE 6: Expression of signaling pathway protein in HepG2 cells. ${ }^{*} P<0.05$ versus Control group.

important cell adhesion factor, participates in and mediates the adhesion between cells and is inextricably linked to the invasion and metastasis of various tumors. Its low expression or loss of expression plays a key role in epithelial mesenchymal transition [16]. Studies have shown that [17] overexpression of TCF4 in canine kidney epithelial cells can increase cell invasive ability, thereby accelerating the occurrence of tumor EMT, which can be represented by remarkable downregulation of epithelial marker protein E-cadherin and upregulation of the interstitial marker protein vimentin. To further explore the molecular mechanism of the effect of Codonopsis pilosula polysaccharide on the motility of hepatocellular carcinoma cells cultured in vitro, we performed Western blot and found that Codonopsis pilosula polysaccharidetreated cells showed higher expression of epithelial marker protein E-cadherin but lower expression of interstitial marker protein $\mathrm{N}$-cadherin and migration marker protein VEGF. The results indicated that Codonopsis pilosula polysaccharide can inhibit the loss of polarity between human hepatoma HepG2 cells cultured in vitro, slow the development of stromal cells, and reduce the invasion and metastasis ability of cells, thus delaying the occurrence of EMT in HepG2 cells.

Wnt signaling pathway is involved in tumor formation. Upon activation, $\beta$-catenin translocates into the nucleus to competitively bind with TCF 4 to form $\beta$-catenin/TCF 4 complex. The complex then activates downstream transcription factors of TCF4, such as cycD and c-Myc, which ultimately leads to cell abnormalities, proliferation, and tumorigenesis $[18,19]$. Studies have shown that abnormal activation of the TCF4 target gene by Wnt signaling can promote malignant transformation of colorectal cancer [20], and downregulation of the $\mathrm{Wnt} / \beta$-catenin signaling pathway can inhibit epithelial-mesenchymal transition in nonsmall cell lung cancer [21]. Therefore, in order to investigate the regulatory effect of concentration-dependent Codonopsis pilosula polysaccharide treatment on the signaling pathway $\beta$-catenin/TCF4 and its effect on human hepatoma HepG2 cells cultured in vitro, we performed series of experiments and found that the expression of $\beta$-catenin, TCF4, and the downstream C-Myc protein were significantly inhibited. These results indicated that Codonopsis pilosula polysaccharide can inhibit the proliferation, invasion, and EMT of human hepatoma HepG2 cells by downregulating the $\beta$-catenin/TCF4 signaling pathway.

In summary, this paper explored the role of Codonopsis pilosula polysaccharide in the proliferation, invasion, and interstitial transformation of human hepatoma HepG2 cells cultured in vitro, suggesting that the inhibition of Betacatenin/Tcf4 protein expression by Codonopsis pilosula polysaccharide is related to the growth and metastasis of human hepatoma HepG2 cells cultured in vitro. This paper also provides experimental data for the role of Codonopsis pilosula polysaccharide in the occurrence and development of liver cancer, but there is still a lack of research on the effect of Codonopsis pilosula polysaccharide on cell growth and metastasis and its role in the growth of liver cancer cells in vivo in the presence of pathway inhibitor/activator.

\section{Data Availability}

The data used to support the findings of this study are available from the corresponding author upon request.

\section{Conflicts of Interest}

The authors declare that there is no conflict of interest regarding the publication of this paper. 


\section{Acknowledgments}

This work was supported by Huai'an Science and Technology Project (HAB201723).

\section{References}

[1] M. Sayiner, P. Golabi, and Z. M. Younossi, "Disease burden of hepatocellular carcinoma: a global perspective," Digestive Diseases and Sciences, vol. 64, no. 4, pp. 910-917, 2019.

[2] J. F. Jiang, Y. C. Lao, B. H. Yuan et al., "Treatment of hepatocellular carcinoma with portal vein tumor thrombus: advances and challenges," Oncotarget, vol. 8, no. 20, pp. 3391133921, 2017.

[3] J. Balogh, D. Victor, E. H. Asham et al., "Hepatocellular carcinoma: a review," Journal of Hepatocellular Carcinoma, vol. 3 , pp. 41-53, 2016.

[4] P. Zhang, L. Hu, R. Bai et al., "Structural characterization of a pectic polysaccharide from Codonopsis pilosula and its immunomodulatory activities in vivo and in vitro," International Journal of Biological Macromolecules, vol. 104, Pt A, pp. 1359-1369, 2017.

[5] C. Liu, J. Chen, E. Li et al., "Solomonseal polysaccharide and sulfated Codonopsis pilosula polysaccharide synergistically resist Newcastle disease virus," PLoS One, vol. 10, no. 2, article e0117916, 2015.

[6] C. Liu, J. Chen, E. Li et al., “The comparison of antioxidative and hepatoprotective activities of Codonopsis pilosula polysaccharide (CP) and sulfated CP," International Immunopharmacology, vol. 24, no. 2, pp. 299-305, 2015.

[7] X. Chu, X. J. Liu, J. M. Qiu, X. L. Zeng, and H. R. Bao, "Inhibitory effects of codonopsis pilosula polysaccharides on the deterioration of impaired phagocytosis of alveolar macrophage induced by fine particulate matter in chronic obstructive pulmonary disease mice," Zhonghua Yi Xue Za Zhi, vol. 96, no. 14, pp. 1134-1138, 2016.

[8] X. Chu, X. J. Liu, J. M. Qiu, X. L. Zeng, H. R. Bao, and J. Shu, "Effects of Astragalus and Codonopsis pilosula polysaccharides on alveolar macrophage phagocytosis and inflammation in chronic obstructive pulmonary disease mice exposed to PM2.5," Environmental Toxicology and Pharmacology, vol. 48, pp. 76-84, 2016.

[9] Q. Zhang, Y. Xia, H. Luo et al., “Codonopsis pilosula polysaccharide attenuates tau hyperphosphorylation and cognitive impairments in hTau infected mice," Frontiers in Molecular Neuroscience, vol. 11, p. 437, 2018.

[10] M. Chen, Y. Li, Z. Liu et al., "Exopolysaccharides from a Codonopsis pilosula endophyte activate macrophages and inhibit cancer cell proliferation and migration," Thoracic Cancer, vol. 9, no. 5, pp. 630-639, 2018.

[11] T. Xin, F. Zhang, Q. Jiang et al., "The inhibitory effect of a polysaccharide from Codonopsis pilosula on tumor growth and metastasis in vitro," International Journal of Biological Macromolecules, vol. 51, no. 5, pp. 788-793, 2012.

[12] B. H. Yuan, W. P. Yuan, R. H. Li et al., "Propensity scorebased comparison of hepatic resection and transarterial chemoembolization for patients with advanced hepatocellular carcinoma," Tumour Biology, vol. 37, no. 2, pp. 24352441, 2016.

[13] R. Bai, W. Li, Y. Li et al., "Cytotoxicity of two water-soluble polysaccharides from Codonopsis pilosula Nannf. var. modesta (Nannf.) L.T.Shen against human hepatocellular carcinoma HepG2 cells and its mechanism," International Journal of Biological Macromolecules, vol. 120, pp. 15441550, 2018.

[14] J. Li, R. Yang, Y. Dong, M. Chen, Y. Wang, and G. Wang, "Knockdown of FOXO3a induces epithelial- mesenchymal transition and promotes metastasis of pancreatic ductal adenocarcinoma by activation of the $\beta$-catenin/TCF 4 pathway through SPRY2," Journal of Experimental \& Clinical Cancer Research, vol. 38, no. 1, p. 38, 2019.

[15] L. Przybyla, J. M. Muncie, and V. M. Weaver, "Mechanical control of epithelial-to-mesenchymal transitions in development and cancer," Annual Review of Cell and Developmental Biology, vol. 32, no. 1, pp. 527-554, 2016.

[16] C. Xiangming, S. Hokita, K. Nuruki et al., "The expression of cadherin-catenin complex in association with the clinicopathologic features of early gastric cancer," Surgery Today, vol. 28, no. 6, pp. 587-594, 1998.

[17] V. R. Sobrado, G. Moreno-Bueno, E. Cubillo et al., "The class I bHLH factors E2-2A and E2-2B regulate EMT," Journal of Cell Science, vol. 122, no. 7, pp. 1014-1024, 2009.

[18] T. Valenta, G. Hausmann, and K. Basler, "The many faces and functions of $\beta$-catenin," The EMBO Journal, vol. 31, no. 12, pp. 2714-2736, 2012.

[19] A. S. Arboatti, F. Lambertucci, M. G. Sedlmeier et al., "Diethylnitrosamine enhances hepatic tumorigenic pathways in mice fed with high fat diet (Hfd)," Chemico-Biological Interactions, vol. 303, pp. 70-78, 2019.

[20] Y. Yao, J. Zuo, and Y. Wei, "Targeting of TRX2 by miR-330-3p in melanoma inhibits proliferation," Biomedicine \& Pharmacotherapy, vol. 107, pp. 1020-1029, 2018.

[21] J. Cai, L. Fang, Y. Huang et al., "Simultaneous overactivation of Wnt $/ \beta$-catenin and TGF $\beta$ signalling by miR-128-3p confers chemoresistance-associated metastasis in NSCLC," Nature Communications, vol. 8, p. 15870, 2017. 


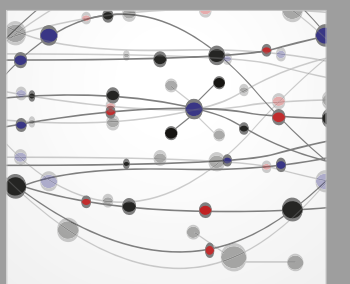

The Scientific World Journal
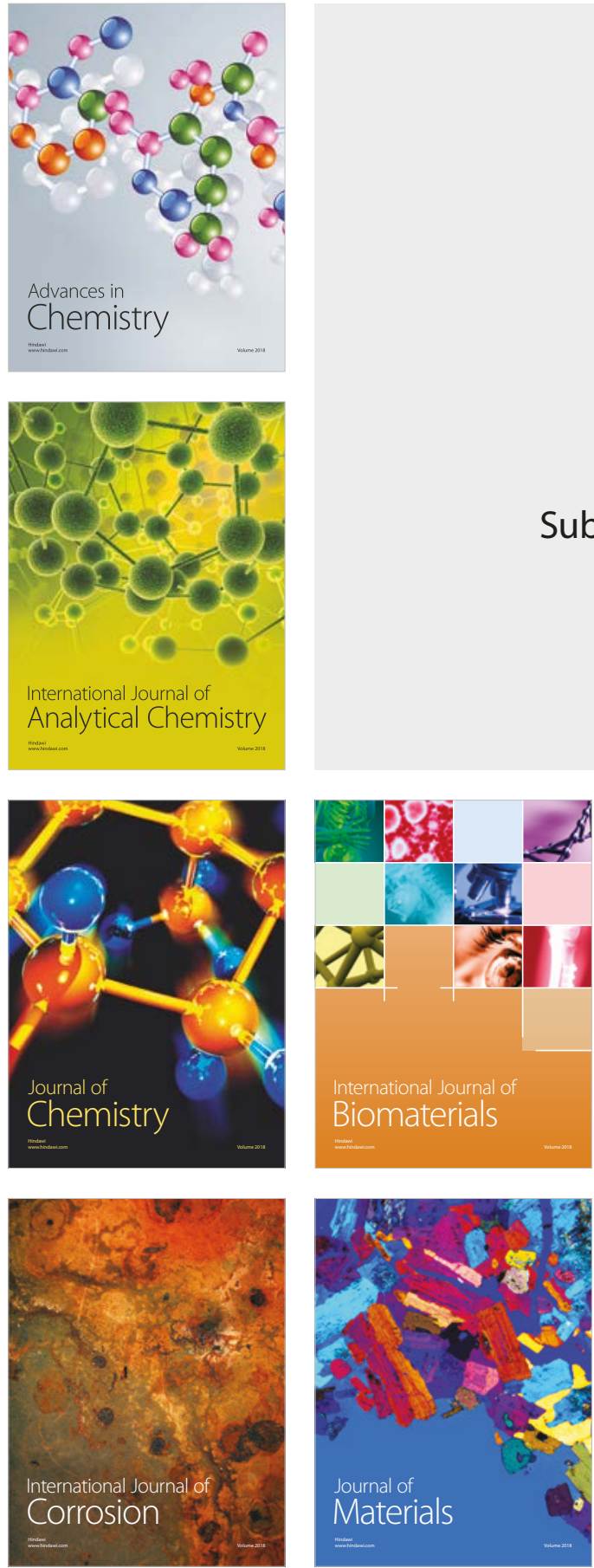

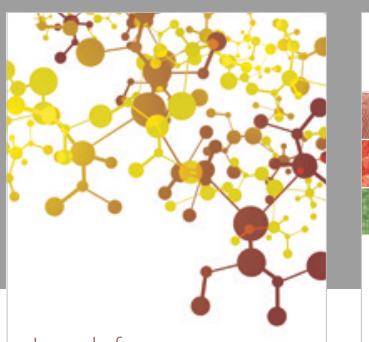

Journal of

Applied Chemistry
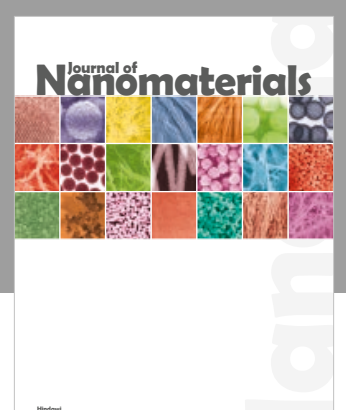

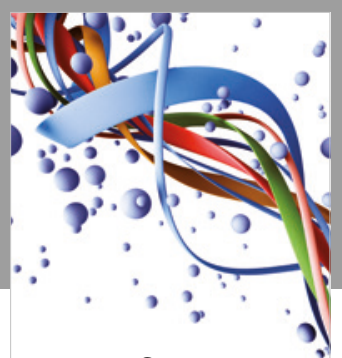

Scientifica

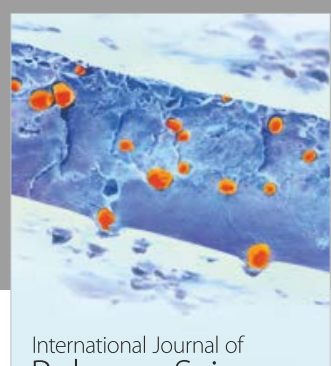

Polymer Science

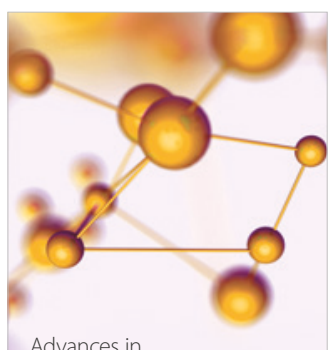

Physical Chemistry
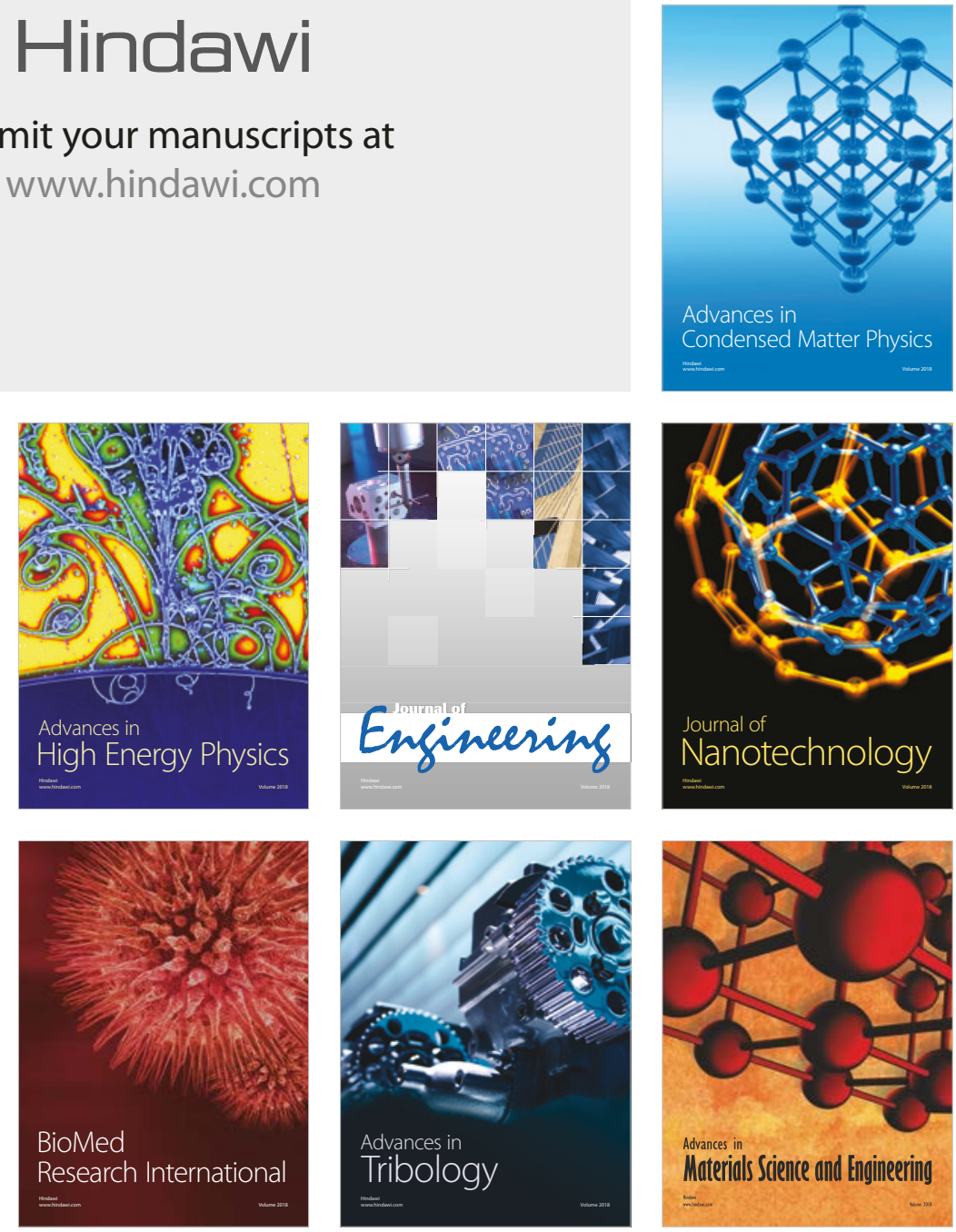